\title{
EVENTOS ADVERSOS E O CUIDADO SEGURO DE ENFERMAGEM NA ATENÇÃO PRIMÁRIA À SAÚDE: REVISÃO INTEGRATIVA
}

\section{ADVERSE EVENTS AND SAFE NURSING CARE IN PRIMARY HEALTH CARE: INTEGRATIVE REVIEW}

\author{
Bruna Carine Heinrichs $^{1} *$ Ana Maria Cisotto Weihermann ${ }^{2} *$ Rosana Amora Ascari $^{3}$
}

\begin{abstract}
RESUMO
Objetivo: Mapear o que a produção científica aborda sobre eventos adversos relacionados à prática profissional de enfermagem brasileira na atenção primária à saúde. Metodologia: Trata-se de um estudo de revisão integrativa da literatura na Biblioteca Virtual em Saúde, através dos descritores "Eventos adversos", "Segurança do paciente", "Enfermagem" e "Atenção Primária à Saúde", realizada no período compreendido entre janeiro de 2011 e setembro de 2020. Das 675 publicações elegíveis analisadas, dez foram incluídas por atenderem ao objetivo. Resultados: Da análise dos artigos emergiram quatro categorias, a saber: Tipos de eventos adversos; Fatores relacionados aos eventos adversos; Notificação de eventos adversos; Estratégias de gestão frente aos eventos adversos. Conclusão: Os eventos adversos estão presentes na Atenção Primária à Saúde e sua notificação deve ser reforçada. Faz-se necessário investir em estratégias de gestão, apoiar a capacitação profissional, a comunicação efetiva e a cultura de segurança do paciente, para minimizar os eventos adversos que permeiam a assistência à saúde.
\end{abstract}

Palavras-Chave: Eventos Adversos; Atenção Primária à Saúde; Segurança do Paciente; Enfermagem

\begin{abstract}
Objective: To map what scientific production adresses about adverse events related to Brazilian nursing professional practice in primary health care. Methodology: This is na integrative literature review study in the Virtual Health Library, using the descriptors "Adverse events", "Patient safety", "Nursing" and "Primary Health Care", from January 2011 to September 2020. Of the 675 eligible publications analyzed, ten were included for meeting the objective. Results: From the analisis of the articles, four categories emerged, namely: Types of adverse events; Factors related to adverse events; Notification of adverse events; Management Strategies in the face of adverse events. Conclusion: Adverse events are present in primary health care and their notification must be reinforced. It is necessary to invest in management strategies, support professional training, effective communication and a culture of patient safety to minimize adverse events that permeate health care.
\end{abstract}

Keywords: Adverse Events; Primary Health Care; Patient Safety; Nursing.

\footnotetext{
${ }^{1}$ Acadêmica de Enfermagem na Universidade do Contestado (UnC). Email: brunacarineheinrichs@gmail.com

${ }^{2}$ Enfermeira. Mestre em Enfermagem pela Universidade Federal de santa Catarina (UFSC). Docente na Universidade do Contestado (UnC). E-mail: anamaria@ unc.br. ORCID: https://orcid.org/0000-0002-6596-1406

${ }^{3}$ Doutora em Enfermagem pela Universidade Federal do Rio Grande do Sul (UFRGS). Docente do Departamento de Enfermagem e do Mestrado Profissional em Enfermagem na Atenção Primária à Saúde na Universidade do Estado de Santa Catarina (UDESC). Docente das Residências em Saúde da Associação Hospitalar Lenoir Vargas Ferreira/Hospital Regional do Oeste (AHLVF/HRO). Email: rosana.ascari@udesc.br. ORCID: https://orcid.org/0000-0002-2281-8642
} 


\section{INTRODUÇÃO}

A segurança do paciente tem sido considerada um dos pilares que sustentam os níveis da qualidade dos serviços de saúde, sendo identificada como de grande impacto nas discussões envolvendo a qualidade da assistência. Os riscos de eventos adversos (EA) existem em diferentes cenários assistenciais, com maior ênfase na literatura científica para a área hospitalar ${ }^{(2-3)}$, provavelmente em decurso do risco da ocorrência de EA estar associado ao nível de complexidade do serviço de saúde ${ }^{(4)}$, uma vez que, quanto maior e mais complexos são os serviços de saúde oferecidos, maiores são os riscos de erros e $\mathrm{EA}^{(5)}$.

Estudo realizado em instituições de ensino de enfermagem, acerca da percepção dos EA, sinalizou que a sobrecarga de trabalho e a demanda de cuidados contribuem para o risco de ocorrência de algum $\mathrm{EA}^{(6)}$. Pela prática profissional percebe-se escassa discussão acerca dos EA na Atenção Primária à Saúde (APS), o que favorece que tais eventos passem despercebidos e, por vezes, não notificados. No entanto, nem todos os erros culminam em EA e nem todos os EA são resultantes de $\operatorname{erros}^{(5)}$. Nesse sentido, a segurança do paciente apresenta íntima relação com a excelência almejada no desenvolvimento dos cuidados de enfermagem e nos serviços de saúde dispensados à população brasileira, sobretudo na rede pública de saúde.

Pesquisadores que investigaram os motivos para a não notificação de incidentes de segurança do paciente por profissionais de saúde, sinalizam para a ocorrência de subnotificação decorrente do medo de notificar, bem como pela falta de conhecimento dos procedimentos necessários para a notificação, além da centralização da notificação no profissional enfermeiro e de notificações centradas em eventos mais graves. Embora os EA sejam, por vezes, associados ao erro humano, deve-se considerar as condições estruturais e organizacionais envolvidas nas atividades laborais, bem como a qualificação profissional $^{(7)}$.

Importante considerar que as ferramentas de notificação promovem a qualidade e a cultura de segurança, visando sempre o aprendizado para a garantia da promoção e prevenção da saúde da população e servindo de importante ferramenta de vigilância sanitária para a gestão dos serviços de saúde, de modo a fortalecer a tomada de decisão frente aos incidentes relacionados à segurança do paciente ${ }^{(7-9)}$.

Os profissionais de enfermagem, juntamente com o gestor da unidade, têm entendimento da real necessidade de 
treinamentos, capacitações, educação permanente e reuniões em equipe, com objetivo de garantir a prevenção de agravos e qualificar a assistência à saúde ${ }^{(4)}$.

Apesar dos avanços no âmbito da segurança do paciente, a falta de compreensão e conhecimento dos profissionais face ao erro tem destaque em noticiários da mídia social, causando grande impacto social, exigindo esforços, de todo o sistema de saúde, no sentido de prestar uma assitência com profissionais participativos e atentos para o processo de identificação e prevenção, garantindo, assim, menores índices de $\mathrm{EA}^{(5)}$.

Portanto, as ações e atitudes frente aos EA devem propiciar a atenuação dos danos e riscos inerentes ao paciente, visto que o aumento da resiliência no âmbito organizacional garante um processo de cuidar mais seguro ao paciente ${ }^{(9)}$.

Mediante o exposto, considerando a importância do reconhecimento dos EA e seus fatores contribuintes, bem como as estratégias de gestão na atenção primária à saúde para mitigar a ocorrência de EA, questiona-se: o que a produção científica brasileira aborda sobre eventos adversos relacionados à prática de enfermagem na APS? Buscou-se, portanto, através da pesquisa, mapear o que a produção científica aborda sobre eventos adversos relacionados à prática profissional de enfermagem brasileira na APS.

\section{MATERIAL E MÉTODOS}

Trata-se de uma revisão integrativa da literatura, um método que reúne, avalia e sintetiza os resultados de pesquisas sobre uma temática específica. Neste estudo foram percorridas as seguintes etapas: (1) definição do tema de pesquisa com elaboração da questão norteadora; (2) definição dos critérios de inclusão e exclusão de estudos; definição das informações a serem extraídas dos estudos selecionados; (4) análise dos estudos com decodificação e categorização dos achados; (5) análise e interpretação dos resultados; (6) apresentação do relatório de pesquisa(Erro! Fonte de referência não encontrada. $^{-11)}$.

Para a elaboração da questão de pesquisa utilizou-se a estratégia PICO, que representa um acrônimo para: (P) Paciente, (I) Intervenção, (C) Comparação, e "Outcomes" (desfecho). O uso desses quatro componentes possibilita formular a questão de pesquisa, além da identificação de palavraschave que auxiliam na localização de estudos primários nas bases de dados Erro! Fonte de referência não encontrada.). Assim, a questão de pesquisa delimitada foi: o que a produção científica brasileira aborda sobre eventos adversos relacionados à prática profissional de enfermagem na atenção primária à saúde? Nela, o primeiro elemento 
da estratégia $(\mathrm{P})$ consiste no profissional de enfermagem da APS no Brasil; o segundo (I), os EA; e o quarto elemento $(\mathrm{O})$ que tipo de EA ocorre na APS, fatores relacionados, notificações e estratégias de gestão frente os EA na APS. Ressalta-se que, dependendo do método de revisão, não se emprega todos os elementos da estratégia PICO, o que justifica a não utilização do terceiro elemento (C comparação) nesta revisão.

A busca dos estudos primários ocorreu no período compreendido entre os dias 24 e 26 de outubro de 2020, realizada na Biblioteca Virtual em Saúde (BVS), a qual contempla as bases de dados da Literatura Latino-americana e do Caribe em Ciências da Saúde (LILACS), Medical Literature Analysis and Retrieval System Online (MEDLINE) e Base de dados em Enfermagem (BDENF), entre outras, utilizando-se a associação dos descritores em saúde (http://decs.bvs.br), ou unitermo em dois cruzamentos específicos: "Eventos adversos" AND "Atenção Primária à Saúde" e, "Eventos adversos" AND "Enfermagem", com auxílio do Programa Academical $^{(13)}$.

Considerou-se critério de inclusão dos estudos: artigos originais que abordavam os EA relacionados à prática profissional de enfermagem na APS no Brasil, disponíveis em formato completo, eletronicamente, publicados no período compreendido entre os meses de janeiro de 2011 e setembro de 2020 , escritos nos idiomas português, inglês e espanhol. Foram excluídos estudos de revisões de literatura, estudos secundários, tais como revisão sistemática, carta-resposta, reflexões e editoriais, artigos duplicados e artigos envolvendo EA de medicações específicas. A coleta de dados foi realizada no mês de outubro de 2020.

Como estratégias de busca foram aplicados os filtros de: temporalidade, idioma e disponibilidade dos artigos em texto completo; e utilizando os cruzamentos de descritores e unitermos no idioma em português, foram identificados 675 artigos elegíveis, os quais foram importados para software Academical. Por meio do Programa Academical foi possível a extratificação do número de artigos por base de dados disponível na BVS, sendo: LILACS (227 artigos), MEDLINE (315 artigos), BDENF (46 artigos) e IBECS (50 artigos). Seguiu-se a leitura criteriosa de títulos e resumos, aplicando os critérios de seleção previamente definidos, sendo excluídos 661 artigos, de modo que 14 artigos passaram para a segunda etapa, para leitura do texto na íntegra. Foram excluídos, nessa etapa, dois artigos por duplicidade, um artigo por tratar-se de estudo na área hospitalar e um artigo que tinha como cenário outro país. Assim, dez artigos compuseram esta revisão integrativa, 
Figura 1 - Fluxograma de seleção dos artigos gerado pelo Programa Academical. Concórdia - SC, Brasil, 2020

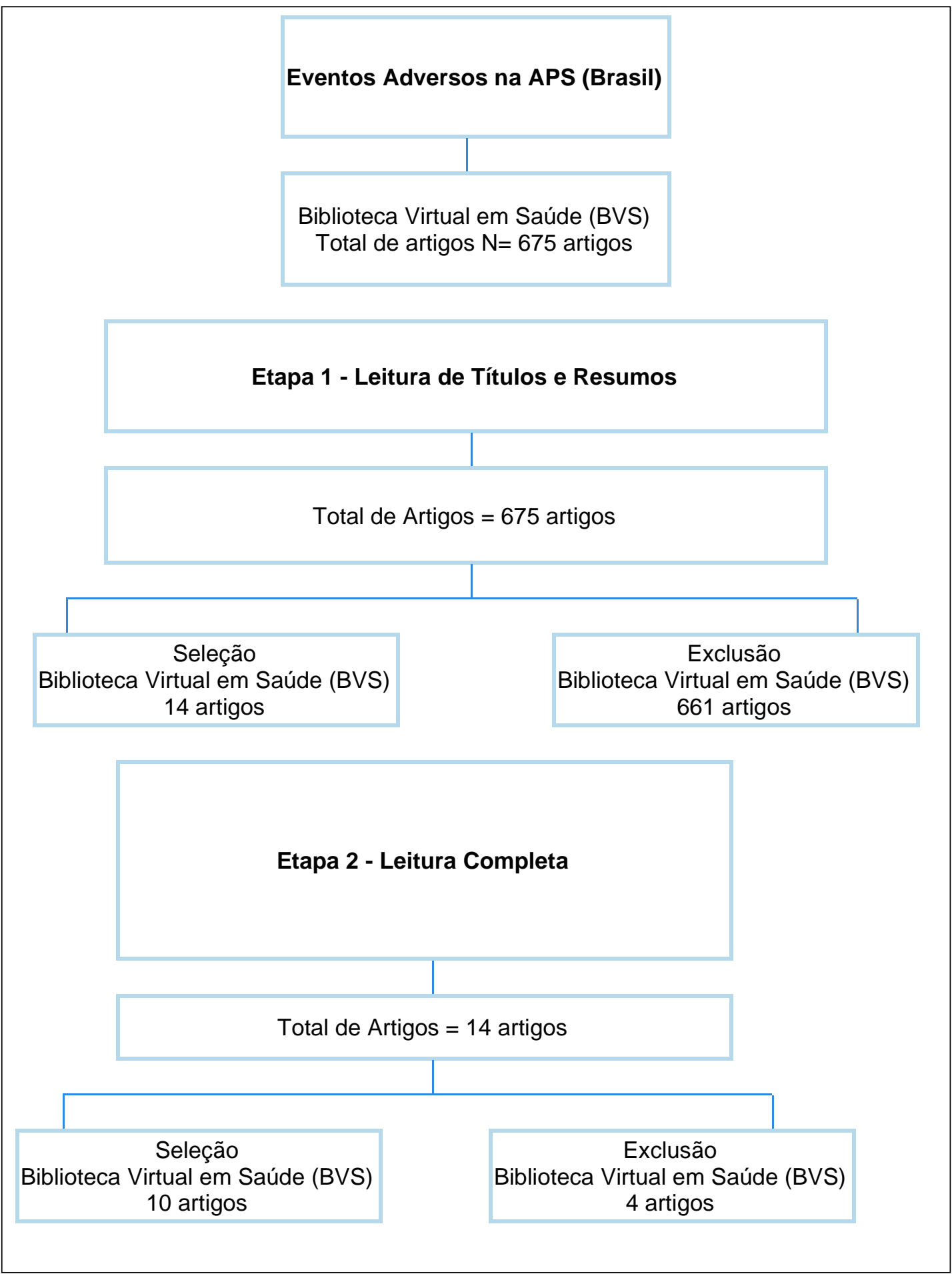

Fonte: Programa Academical, out. 2020. 
integrativa, de acordo com o PRISMA,

A figura 2 apresenta o fluxograma do seguindo os critérios de identificação, triagem, elegibilidade e inclusão(14).

processo de seleção dos artigos a revisão

Figura 2 - Fluxograma do processo de seleção de acordo com o PRISMA. Concórdia - SC, Brasil 2020.

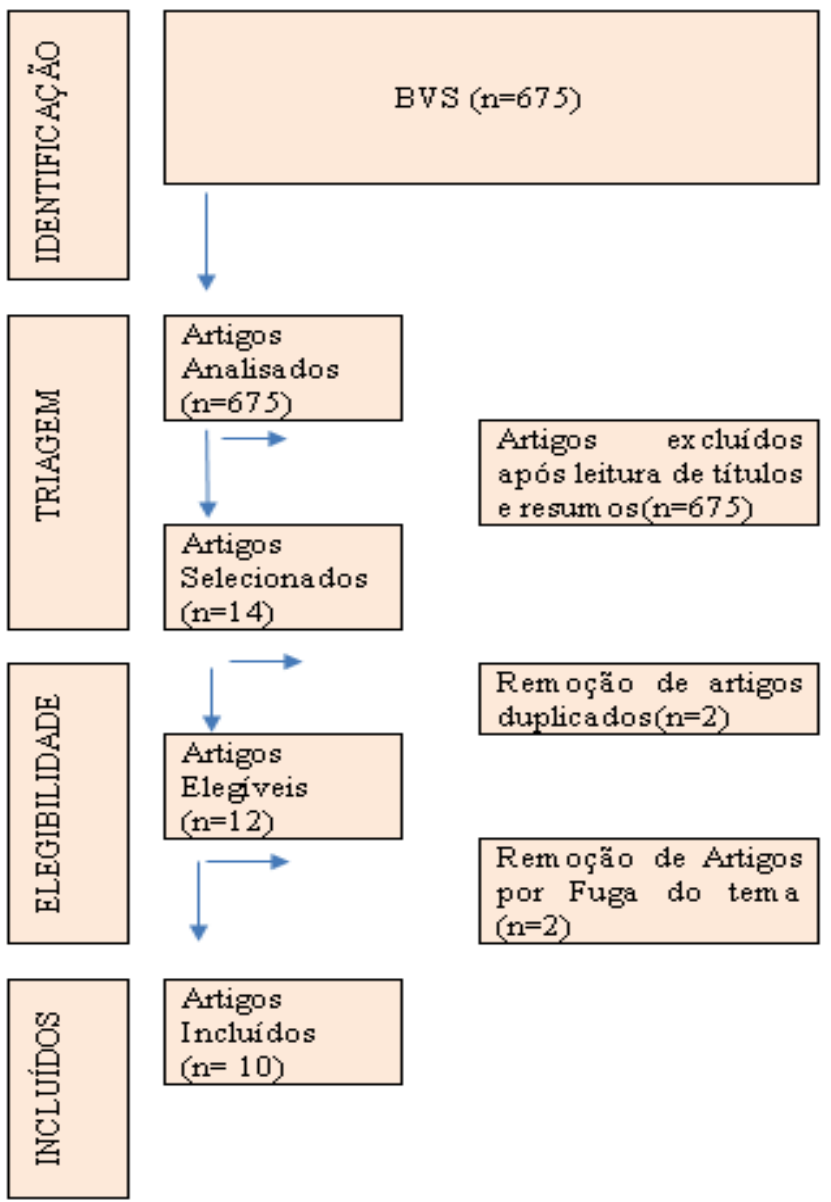

Fonte: Banco de dados dos Autores, 2020, com base em Moher, 2009 ${ }^{(14)}$

A análise dos estudos inclusos na revisão foi realizada com preenchimento de formulário específico para a interpretação dos dados. Para a apresentação dos resultados foi utilizada a estatística simples e os dados foram descritos a partir da tabela de revisão integrativa (Tabela 1). Na sequência, os artigos foram analisados pela técnica de Análise Temática, proposta por Bardin'Erro! Fonte de referência não encontrada.', a qual é 
composta por três etapas distintas: préanálise; a exploração do material e tratamento dos resultados, inferência e interpretação. $\mathrm{O}$ processo de análise deu origem a quatro categorias temáticas, sendo elas: Tipos de EA; Fatores causais relacionados aos EA; Notificação de EA; Estratégias de gestão frente aos EA.

\section{RESULTADOS}

Fundamentado na bibliometria, ou seja, que quantifica, descreve e fornece prognósticos relacionados ao processo de comunicação e escrita ${ }^{(15)}$, foram analisados os aspectos quantitativos dos estudos que abordassem informações sobre EA relacionados à prática profissional de enfermagem na APS, sendo que a amostra final é representada por dez (10) artigos, os quais foram identificados com a letra " $\mathrm{A}$ " de artigo, seguido de número arábico sequencial de 01 à 10 e referenciados por numeração arábica sobrescrita, conforme apresentados na Tabela 1.

Tabela 1. Caracterização da produção científica acerca dos eventos adversos na atenção primária à saúde no período de 2011 a 2020

\begin{tabular}{|c|c|c|c|c|c|c|c|}
\hline $\mathbf{N}^{\mathbf{O}}$ & Título & Objetivo & Autores & Base & $\begin{array}{c}\text { Publicaçã } \\
\text { o }\end{array}$ & Periódico & $\begin{array}{l}\text { Nível de } \\
\text { Evidência }\end{array}$ \\
\hline $\mathrm{A} 01^{(17)}$ & $\begin{array}{l}\text { Característic } \\
\text { as dos } \\
\text { eventos } \\
\text { adversos na } \\
\text { atenção } \\
\text { primária à } \\
\text { saúde no } \\
\text { Brasil }\end{array}$ & $\begin{array}{l}\text { Avaliar a ocorrência } \\
\text { de incidentes no cuidado à } \\
\text { saúde ao paciente na } \\
\text { atenção primária } \\
\text { brasileira. }\end{array}$ & $\begin{array}{l}\text { Marchon } \\
\text { SG, } \\
\text { Mendes } \\
\text { Junior WV, } \\
\text { Pavão ALB }\end{array}$ & LILACS & $2015 / 11$ & $\begin{array}{l}\text { Cad. } \\
\text { saúde } \\
\text { pública }\end{array}$ & 4 \\
\hline $\mathrm{A} 02^{(18)}$ & $\begin{array}{l}\text { Eventos } \\
\text { adversos } \\
\text { pós- } \\
\text { vacinação: } \\
\text { educação } \\
\text { permanente } \\
\text { para a equipe } \\
\text { de } \\
\text { enfermagem }\end{array}$ & $\begin{array}{l}\text { Realizar ações de } \\
\text { Educação Permanente aos } \\
\text { profissionais de } \\
\text { enfermagem do município } \\
\text { de Guarapuava - PR, } \\
\text { visando à diminuição de } \\
\text { erros na administração de } \\
\text { vacinas e possíveis dos } \\
\text { Eventos Adversos Pós- } \\
\text { Vacinação. }\end{array}$ & $\begin{array}{l}\text { Ternopolsk } \\
\text { i CA, } \\
\text { Baratieri T, } \\
\text { Lenstck } \\
\text { MH }\end{array}$ & LILACS & $2015 / 12$ & $\begin{array}{l}\text { Espaç. } \\
\text { Saúde } \\
\text { (Online) }\end{array}$ & 4 \\
\hline $\mathrm{A} 03^{(19)}$ & $\begin{array}{l}\text { Ocorrência } \\
\text { de eventos } \\
\text { adversos } \\
\text { pós- } \\
\text { vacinação } \\
\text { em idosos }\end{array}$ & $\begin{array}{l}\text { Analisar a ocorrência de } \\
\text { Eventos Adversos Pós- } \\
\text { Vacinação em idosos, no } \\
\text { Brasil, de } 2004 \text { a } 2013 .\end{array}$ & $\begin{array}{l}\text { Linheira- } \\
\text { Bisetto LH, } \\
\text { Ciosa SI, } \\
\text { Cordeiro } \\
\text { TLR, } \\
\text { Boing MS }\end{array}$ & LILACS & $2016 / 12$ & $\begin{array}{l}\text { Cogitare } \\
\text { enferm }\end{array}$ & 4 \\
\hline
\end{tabular}




\begin{tabular}{|c|c|c|c|c|c|c|}
\hline $\mathrm{A} 04^{(20)}$ & $\begin{array}{l}\text { Cuidados } \\
\text { prestados } \\
\text { pela equipe } \\
\text { de } \\
\text { enfermagem } \\
\text { no } \\
\text { atendimento } \\
\text { aos eventos } \\
\text { adversos } \\
\text { pós-vacinais }\end{array}$ & $\begin{array}{l}\text { Identificar os cuidados } \\
\text { prestados pela equipe de } \\
\text { enfermagem no } \\
\text { atendimento aos eventos } \\
\text { adversos } \\
\text { pós-vacinais (EAPV). }\end{array}$ & $\begin{array}{l}\text { Oliveira ES } \\
\text { de A, } \\
\text { Casarin ST, } \\
\text { Ceolin T, } \\
\text { Soares DC }\end{array}$ & BDENF & 2016/04 & $\begin{array}{l}\text { Rev. } \\
\text { Enferm. } \\
\text { UFPI }\end{array}$ \\
\hline $\mathrm{A} 05^{(21)}$ & $\begin{array}{l}\text { Percepção de } \\
\text { Equipes de } \\
\text { Saúde da } \\
\text { Família } \\
\text { sobre a } \\
\text { notificação } \\
\text { de eventos } \\
\text { adversos a } \\
\text { medicamento } \\
\text { s }\end{array}$ & $\begin{array}{l}\text { Avaliar se profissionais } \\
\text { de saúde da equipe de } \\
\text { saúde da família } \\
\text { reconhecem a notificação } \\
\text { de eventos adversos a } \\
\text { medicamentos (EAM) } \\
\text { como forma de promoção } \\
\text { a Segurança do Paciente e } \\
\text { as possíveis barreiras para } \\
\text { execução da mesma }\end{array}$ & $\begin{array}{l}\text { Pereira LB, } \\
\text { Jobim L, } \\
\text { Bueno D }\end{array}$ & LILACS & 2018/08 & $\begin{array}{l}\text { Saúde } \\
\text { Redes }\end{array}$ \\
\hline $\mathrm{A} 06^{(22)}$ & $\begin{array}{l}\text { Eventos } \\
\text { adversos } \\
\text { ocasionados } \\
\text { pela } \\
\text { assistência } \\
\text { de } \\
\text { enfermagem: } \\
\text { noticiados } \\
\text { pela mídia }\end{array}$ & $\begin{array}{l}\text { Caracterizar as } \\
\text { informações de } \\
\text { documentos audiovisuais } \\
\text { disponíveis no YouTube } \\
\text { sobre os eventos adversos } \\
\text { ocasionados pela } \\
\text { assistência de } \\
\text { enfermagem no Brasil e } \\
\text { noticiados pela mídia } \\
\text { televisiva }\end{array}$ & $\begin{array}{l}\text { Moura R } \\
\text { dos S, } \\
\text { Saraiva } \\
\text { FJC, Santos } \\
\text { RM dos, } \\
\text { Santos RF } \\
\text { de M dos, } \\
\text { Rocha KR } \\
\text { da SL, } \\
\text { Virgem } \\
\text { MRC da }\end{array}$ & BDENF & 2018/06 & $\begin{array}{l}\text { Rev. } \\
\text { Enferm. } \\
\text { UFPE on } \\
\text { line }\end{array}$ \\
\hline $\mathrm{A} 07^{(23)}$ & $\begin{array}{l}\text { A prática da } \\
\text { enfermagem } \\
\text { frente aos } \\
\text { eventos } \\
\text { adversos } \\
\text { pós- } \\
\text { vacinação. }\end{array}$ & $\begin{array}{l}\text { Identificar eventos } \\
\text { adversos pós-vacinação, } \\
\text { foco da prática da } \\
\text { enfermagem, em base de } \\
\text { dados do Sistema de } \\
\text { Informação de Eventos } \\
\text { Adversos Pós-Vacinação } \\
\text { e discutir a atuação do } \\
\text { enfermeiro na sua } \\
\text { vigilância. }\end{array}$ & $\begin{array}{l}\text { Bisetto } \\
\text { LHL, } \\
\text { Cubas MR, } \\
\text { Malucelli A }\end{array}$ & $\begin{array}{l}\text { MEDLIN } \\
\text { E }\end{array}$ & $2011 / 10$ & $\begin{array}{l}\text { Rev Esc } \\
\text { Enferm } \\
\text { USP }\end{array}$ \\
\hline $\mathrm{A} 08^{(24)}$ & $\begin{array}{l}\text { Manejo de } \\
\text { eventos } \\
\text { adversos } \\
\text { pós- } \\
\text { vacinação } \\
\text { pela equipe } \\
\text { de } \\
\text { enfermagem: } \\
\text { desafios para } \\
\text { o cuidado }\end{array}$ & $\begin{array}{l}\text { Analisar como a equipe } \\
\text { de enfermagem de um } \\
\text { centro de especialidades } \\
\text { desenvolve as práticas de } \\
\text { cuidado relacionadas ao } \\
\text { manejo de EAPV leves, } \\
\text { moderados e graves entre } \\
\text { crianças de até um ano de } \\
\text { idade }\end{array}$ & $\begin{array}{l}\text { Alves H, } \\
\text { Domingos } \\
\text { LMG }\end{array}$ & LILACS & $2013 / 12$ & $\begin{array}{l}\text { Rev. } \\
\text { Enferm. } \\
\text { UERJ }\end{array}$ \\
\hline $\mathrm{A} 09^{(25)}$ & Análise de & Analisar os vídeos do & Salvador & LILACS & $2014 / 12$ & REME \\
\hline
\end{tabular}


vídeos do YouTube sobre eventos youtube

sobre

eventos

adversos em

saúde

A10 (26) Análise da ocorrência de evento adverso pósvacinação decorrente de erro de imunização adversos em saúde, de relações com a segurança do paciente.

Analisar a ocorrência de Evento Adverso PósVacinação (EAPV) decorrente de erro de 2003 a 2013.
PTC de O, Martins rev. min. identificando a existência

CCF, Alves

KYA,

Costa TD da, Santos VEP imunização, no Paraná, de

Bisetto

LILACS 2017/02 Rev.

enferm

LHL,

Ciosak SI

Brás.

Enferm

Fonte: Banco de dados dos Autores (2020).

Ao analisar as características dos artigos, identificou-se que o maior número de artigos foram publicados nos anos de $2015^{(17,0)}, 2016^{(19,20)}$ e $2018^{(21,0)}$ e nenhum artigo foi selecionado nos anos de 2012, 2019 e 2020. Identificaram-se também publicações nos anos de $2011^{(23)}, 2013^{(24)}, 2014^{(25)}$ e $2017^{(26)}$ com uma publicação por ano. Os estudos foram publicados em diferentes periódicos: Caderno de Saúde Pública(17), Revista Espaço para a Saúde ${ }^{(18)}$, Cogitare Enfermagem $^{(19)}$, Revista de Enfermagem da UFPI $^{(20)}$, Saúde em Redes ${ }^{(21)}$, Revista de Enfermagem da UFPE ${ }^{(22)}$, Revista da Escola de Enfermagem da USP ${ }^{(23)}$, Revista de Enfermagem da UERJ ${ }^{(24)}$ Revista Minera de Enfermagem $^{(25)}$ e Revista Brasileira de Enfermagem $^{(26)}$, representando um artigo por periódico.
De acordo com a localização geográfica dos estudos, identificou-se que o maior número de estudos derivam da região Sul do país $(60 \%)$, sendo quatro do Paraná ${ }^{(18-}$ 19,23,26) e dois do Rio Grande do Sul ${ }^{(20-21)}$, seguido da região Sudeste (20\%), com dois estudos do estado do Rio de Janeiro ${ }^{(17,24)}$ e região Nordeste (20\%), contemplando os estados de Alagoas $^{(22)}$ e Rio Grande do Norte $^{(25)}$. Destaca-se que quatro estudos abordaram dados de referência nacional ${ }^{(19,22-}$ 23,25).

Em relação ao idioma, todos os artigos foram encontrados no idioma português ${ }^{(21,23)}$, embora alguns tenham sido publicados bilíngue $(70 \%)$ - português/inglês ${ }^{(07,19,25,23)}$.

Quanto ao nível de evidência científica, é possível traçar uma classificação de acordo com a abordagem metodológica empregada nos estudos, seguindo um padrão 
hierárquico. No presente estudo de RI, utilizou-se a classificação de nível de evidência conforme segue: 1 - evidências provenientes de revisão sistemática ou metanálise de todos os ensaios clínicos randomizados, controlados ou oriundas de diretrizes clínicas baseadas em revisões sistemáticas de ensaios clínicos randomizados controlados; 2 - evidências derivadas de pelo menos um ensaio clínico randomizado controlado bem delineado; 3 - evidências obtidas de ensaios clínicos bem delineados sem randomização; 4 - evidências provenientes de estudos de coorte e de caso controle bem delineados; 5 - evidências originárias de revisão sistemática de estudos descritivos e qualitativos; 6 - evidências derivadas de um único estudo descritivo ou qualitativo e 7 - evidências oriundas de opinião de autoridades e/ou relatório de comitês de especialistas $^{(27)}$.

A partir disso, foi possível verificar que, dos artigos incluídos nessa revisão, não houve classificados de acordo com o nível de evidência 1, 2, 3, 5 e 7, sendo três periódicos classificados no nível de evidência $4^{(17-19)} \mathrm{e}$ sete com nível de evidência $6^{(20-26)}$.

Da análise temática dos artigos emergiram quatro categorias, a saber: Tipos de EA; Fatores relacionados aos EA; Notificação de EA; Estratégias de gestão frente aos EA.

\section{Tipos de EA}

Observa-se que $90 \%$ dos estudos abordaram algum tipo de EA. Relacionados à medicação foram dois estudos $(20 \%)^{(21-22)}$, na sua maioria associados a administração de medicamento pela via errada e fármaco errado, como, por exemplo, "a administração de dieta enteral pela via parenteral"(22) ou “administração de ácido em vez de sedativo"(22), dentre esses, é possível ainda mencionar o erro de inserção de cateter periférico para administração de medicação ${ }^{(22)}$.

Nesse sentido, um estudo que caracterizou as informações de documentos audiovisuais disponíveis no YouTube sobre os EA ocasionados pela assistência de enfermagem

no Brasil e noticiados pela mídia televisiva, sinalizou que os títulos dos vídeos eram impactantes, apesar de não revelar conhecimento sobre a diferença conceitual entre erro e EA, chegando a 76.307 $\operatorname{acessos}^{(22)}$. Ainda, houve incapacidade de reconhecer erros relacionados a medicamentos em quase $40 \%$ dos participantes de estudo que avaliou se profissionais de saúde da APS reconhecem a notificação de EV relacionados com medicamentos como forma de promoção da segurança do paciente ${ }^{(21)}$. 
Apenas um estudo abordou erros de diagnóstico ou diagnóstico incorreto que causaram EA e evoluíram para complicações e piora do quadro clínico do paciente, relacionados, na sua maioria, ao descuido do profissional em não realizar corretamente o exame físico, demora na obtenção de informações, interpretação dos achados laboratoriais e desconsideração de exames ${ }^{(07)}$.

Chama a atenção que metade dos estudos dessa revisão estão relacionados a administração de vacinas ${ }^{(18-19,23-24,26)}$, prevalecendo as manifestações locais e sistêmicas, por vacinas virais e bacterianas ${ }^{(18)}$, sendo mais citadas as vacinas: $\mathrm{BCG}^{(24,26)}$, febre amarela ${ }^{(19,24)}$, difteria e tétano, hepatite B, pneumocócica 23 valente, influenza( ${ }^{(19)}$, Tríplice Viral, Tetravalente e Dupla viral ${ }^{(24)}$.

Dentre as manifestações locais causadas pelas vacinas encontrou-se 0 abscesso local ${ }^{(24)}$, abscesso subcutâneo quente e frio, linfadenopatia regional supurada ${ }^{(26)}$, linfadenomegalia não supurada, enduração, exantema generalizado $^{(23-24)}$, nódulo ${ }^{(19,23)}$, dor, calor e rubor, predominantes nas vacinas influenza $(42,64 \%)$ e difteria, tétano $(39,64 \%)$, e a vacina dT foi responsável pelos maiores percentuais de reação de Arthus (70\%), enduração $(53,57 \%)$, dificuldade de deambular $(52,94 \%)$, nódulo $(51,85 \%)$, dor, calor e rubor $(49,95 \%)$ e outras reações locais (edema e outros) $(44,44 \%)^{(19)}$, houve também reações de hipersensibilidade após $2 \mathrm{~h}^{(19,24)}$, com maior prevalência na aplicação da vacina Influenza $^{(19)}$, além desses, foi mencionado a urticária generalizada e as reações sistêmicas como: febre $>38^{\circ} \mathrm{C}$, febre $\geq 39,5^{\circ} \mathrm{C}$, choque anafilático ${ }^{(24)}$ e febre $<39,5^{\circ} \mathrm{C}^{(} 0^{3)}$.

Constatou-se que um artigo abordou EA mais específicos da área hospitalar, porém elencou alguns que estão presentes na APS, sendo: EA devido a complicações decorrentes de quedas e EA a medicações ${ }^{(05)}$.

\section{Fatores causais relacionados aos EA}

Considerando que os serviços de saúde são prestados em ambientes complexos, vários fatores podem contribuir para a ocorrência de EA na APS. As falhas na comunicação com o paciente, comunicação interprofissional e comunicação na rede de atenção são os fatores mais comuns para ocorrência de EA devido a dificuldades para o "bom relacionamento da equipe, as diferenças de opinião, de visão profisssional, de formação acadêmica, de cultura de segurança do paciente, de comportamento, de escolaridade, de hierarquização profissional, e de responsabilização com o paciente"(07:2326).

Dentre as falhas na gestão abordadas nos estudos, estão as relacionadas à falta de remédio na farmácia e receituário apropriado, demora na entrega de exames, falta de 
recursos para adquirir medicamentos, entre outros $^{(17)}$.

Outro fator mencionado é a sobrecarga de trabalho, associada à defasagem salarial do profissional de enfermagem e o ambiente físico inapropriado para o exercício profissional, pois culminam em EA e ao cuidado não seguro do paciente ${ }^{(22)}$.

Os fatores causais de EA relacionados a vacinação estiveram presentes em quatro estudos, associados, na sua maioria, a vacina, aos vacinados, a forma de administração ${ }^{(20)}$, ou devido à prescrição ou indicação de um imunobiológico $^{(19)}$. Isso se deve a alguns fatores, como o desvio das boas práticas de vacinação e o aumento da vigilância de EA pós-vacinação (EAPV), a inquietação no momento da vacinação, principalmente por parte das crianças, e o desconhecimento de alguns profissionais aos tipos de imunobiológicos e a quantidade de doses aplicadas em menores de cinco anos de idade, o que leva a maiores índices de $\mathrm{EAPV}^{(06)}$.

Outro fator importante é a falta de habilidade técnica e de manuseio dos imunobiológicos por parte de alguns vacinadores $^{(18,26)}$, bem como o desconhecimento de alguns profissionais acerca dos $\mathrm{EAPV}^{(18)}$. Há que se considerar, ainda, a estrutura organizacional e o ambiente, fatores que contribuem para a ocorrência de EA, uma vez que, quando esses não apresentam segurança aos usuários, interferem na garantia de uma vacinação segura $^{(06)}$.

Nesse sentido, os estudos sinalizaram aspectos relevantes para a prevenção de EA, sobretudo relacionados à vacinação, tais como a implementação de boas práticas assistenciais e o monitoramento e assistência em casos de EA.

\section{Notificação de EA}

Os fatores relacionados à notificação e subnotificação de EA estiveram presentes em mais da metade dos estudos da RI (70\%). Dentre os fatores relacionados à notificação dos EA, pode-se notar a baixa adesão a essa prática devido a diferentes barreiras, tais como: falta de confiança, medo de exposição por má prática, medo de perda da reputação, o excesso de trabalho e o pouco tempo disponível para realizar a notificação ${ }^{(21)}$, além da escassez de conhecimento de alguns profissionais a respeito da notificação de incidentes $^{(07)}$.

Embora a literatura tenha abordado que a notificação de EAPV é muito importante para a vacinação $\operatorname{segura}^{(19)}$, ainda existem casos de subnotificação ${ }^{(19,24,26)}$. Os motivos da subnotificação compreendem a falta de experiência, que pode dificultar a elucidação do caso, levando a subnotificação do $\mathrm{EAPV}^{(26)}$, e as fragilidades nas ações de 
vigilância epidemiológica $\quad$ e $\quad$ o manejo incorreto dos EAPV pela equipe de enfermagem, especialmente em relação a vacinação infantil ${ }^{(04)}$.

Em um estudo, os profissionais consideram importante notificar apenas os EAPV mais graves e que as notificações não carecem de acompanhamento $^{(18)}$. Porém, em outro estudo, os profissionais acham necessário aprimorar a vigilância sob a notificação dos incidentes como meio de prevenir que tais incidentes possam evoluir para reais $\mathrm{EA}^{(05)}$. Todavia, há que se considerar que o incentivo para notificações de EA no serviço, de forma educativa e sem punições, pode contribuir, de forma significativa, para o fortalecimento da cultura de segurança do paciente, bem como embasar estratégias para implementação de medidas preventivas de novos EA.

\section{Estratégias de gestão frente aos EA}

Percebe-se que grande parte dos estudos analisados apresentaram estratégias de gestão frente à ocorrência de EA. Um dos estudos sugere o fortalecimento da cultura de segurança e do trabalho em equipe entre os profissionais e o envolvimento do paciente na educação profissional, além da mitigação da cultura punitiva, garantindo assim, a qualidade e humanização dos serviços prestados, e a implementação de protocolos, para que a equipe sinta-se confiante na tomada de decisões clínicas e na realização de práticas assistenciais seguras ${ }^{(17)}$.

Outros estudos reconhecem a importância de capacitar os técnicos e discutir com os gestores dos serviços de saúde, estratégias como as campanhas de vacinação para qualificar a vigilância epidemiológica de EAPV $^{(19,26)}$, além de sugerir programas de capacitação profissional e avaliação periódica sobre temática específica, tal como os EAPV $^{(18)}$.

Ainda, estudo sinaliza o uso de ferramentas de promoção da segurança do paciente, no momento da vacinação, como a implementação de orientações referentes às vacinas que serão administradas. O mesmo estudo aborda o uso de compressas frias nas primeiras 24-48 horas para o alívio das reações locais, do banho e do uso de medicamentos para diminuição da temperatura corporal ${ }^{(200)}$.

Outra estratégia sugerida é a qualificação, atualização e desenvolvimento de protocolos clínicos assistencias ${ }^{(04)}$. E a formação de recursos humanos, com implementação da educação continuada nos serviços de saúde ${ }^{(03)}$. Nesse sentido, é importante que os profissionais envolvidos com sala de vacina mantenham-se atualizados e sigam as condutas de vigilância epidemiológica de EA pós-vacinação, 
recomendadas pelo Ministério da Saúde, visando minimizar os impactos à saúde da população, uma vez que EA envolvendo a vacinação foram os que se sobressaíram nessa revisão integrativa.

\section{DISCUSSÃO}

Com o objetivo de proporcionar uma assistência segura ao paciente, os serviços de saúde demonstram considerar indicadores que possam interferir na qualidade assistencial, sendo os EA o indicador mais relevante, pois, embora indesejáveis, os EA são frequentemente observados na prática assistencial, principalmente os relacionados aos erros de medicação(Erro! Fonte de referência não encontrada.), que poderiam ser evitados com cuidados envolvendo a prescrição, armazenamento, dispensação e administração do medicamento ${ }^{(27)}$.

A maior ocorrência de EA está relacionada a vacinação, constituindo uma preocupação cada vez mais constante quando associada, na sua maioria, as semelhanças quanto ao formato e ao tamanho reduzido dos rótulos entre a maioria dos imunobiológicos, o que condiciona o profissional ao erro ${ }^{(27)}$.

Os fatores causais de EA associados à vacinação relacionam-se, na sua maioria, a vacina, aos vacinados, a forma de administração, ou devido à prescrição ou indicação de um imunobiológico. Estudo apresenta dados equivalentes, o qual relata EA decorrentes da inadequação na indicação do imunobiológico, intervalo inadequado entre doses, técnica de administração incorreta, os relacionado ao tipo de imunobiológico utilizado, administração de forma errada, entre outros erros que contribuíram para ocorrência de $\mathrm{EA}^{(0)}$.

Dentre os motivos pelos quais os profissionais não realizam a notificação de EA estão o pouco reconhecimento da segurança do paciente e da importância de notificar um incidente ${ }^{(9)}$.

Contudo, o conhecimento insuficiente de uma pequena quantidade de profissionais sobre o sistema de notificação, o excesso de trabalho, a pressão e a ausência de feedback, são limitações sentidas pelos profissionais das instituições de saúde face à ocorrência de $\mathrm{EA}^{(0)}$.

A literatura aponta que as falhas na comunicação com o paciente, na comunicação interprofissional e na rede de atenção, contribuíram para a ocorrência de $\mathrm{EA}^{(07)}$, e que os profissionais falham na manutenção de uma comunicação efetiva e que favoreça o trabalho em equipe, o que compromete a segurança do paciente ${ }^{(0)}$.

O excesso de trabalho, os déficits na estrutura física e a insatisfação com salários e jornada de trabalho excessiva podem repercutir, de maneira positiva e negativa, na 
qualidade dos cuidados prestados, e, se associadas a condições de trabalho e gestão inapropriada, dificultam a efetividade do serviço da $\operatorname{APS}^{(0)}$.

Promover a segurança do paciente no contexto da APS evidencia a qualidade da assistência prestada, sendo o gestor responsável por incentivar e capacitar os profissionais para a prevenção, notificação e o correto manejo dos possíveis riscos durante a realização da assistência ${ }^{(9)}$.

Ações educativas, como capacitações de equipes, implementação de um sistema de notificação de EA, trabalhar a comunicação interprofissional, melhorar a gestão dos recursos e motivar profissionais para $o$ compartilhamento de dúvidas e acontecimentos como forma de aprendizagem, são formas de auxiliar na prevenção da ocorrência de $\mathrm{EA}^{(4)}$.

\section{CONCLUSÃO}

Os dados evidenciam predomínio de eventos adversos envolvendo medicações e imunizações, tendo como fatores causais a falta de comunicação, falhas na infraestrutura, no dimensionamento de pessoal e na execução da assistência, os quais contribuem para a ocorrência de subnotificações. Aos gestores cabe disponibilizar recursos e ferramentas adequadas, incentivando a equipe na incorporação de boas práticas ao cuidado prestado pela enfermagem na APS.

A notificação de eventos adversos pelos profissionais é permeada por medo, fruto de uma cultura punitiva que pode ser amenizada pela implantação de protocolos clínicos, diálogo entre profissionais e pacientes, educação permanente em saúde e qualificação profissional, tornando possível o reconhecimento e prevenção de eventos adversos em ambiente favorável à melhor segurança do paciente.

De tal modo, a segurança do paciente tem sido o foco principal das instituições de saúde, sendo de suma importância a inserção dessa temática nas discussões em saúde, sobretudo entre profissionais que integram a equipe multiprofissional, com vistas a qualificar a assistência e, consequentemente, minimizar a ocorrência de erros e/ou EA.

Recomenda-se a realização de novos estudos sobre a temática dessa revisão integrativa, envolvendo a implantação de instrumentos de reconhecimento e notificação de EA pelos serviços de saúde de APS e a equipe multiprofissional, favorecendo o diálogo entre profissionais na busca por uma cultura de segurança positiva e não punitiva frente ao fenômeno.

\section{REFERÊNCIAS}


1. Duarte SCM, Stipp MAC, Silva MM, Oliveira

FT. Eventos adversos e segurança na assistência de enfermagem. Rev. Bras. Enferm [Internet]. 2015 Feb [acesso em 23 Out 2020]; 68(1):144-54. Disponível em: http://www.scielo.br/scielo.php?script=sci_a rttext\&pid=S0034-

71672015000100144\&lng=en. DOI : https://doi.org/10.1590/0034-

7167.2015680120p.

2. Figueiredo ML, Oliveira e Silva CS, Brito MFSF, D'Innocenzo M. Analysis of incidents notified in a general hospital. Rev Bras Enferm [Internet]. 2018 [acesso em 23 Out 2020]; 71(1):111-9. Disponível em: https://www.scielo.br/pdf/reben/v71n1/pt_0 034-7167-reben-71-01-0111.pdf. DOI: https://doi.org/10.1590/0034-7167-20160574.

3. Lanzoni GMM, Goularte AF, Koerich C, Reisdorfer E, Miotello M, Meirelles BHS. Eventos adversos e incidentes sem dano em unidades de internação de um hospital especializado em cardiologia. Rev Min Enferm [internet]. 2019 [acesso em 23 out 2020]; 23:e-1184. Disponível em: https://cdn.publisher.gn1.link/reme.org.br/pd f/e1184.pdf. $\quad$ DOI: $10.5935 / 1415-$ 2762.20190032.

4. Santos PVM, Mendes PM, Abreu IM, Sá AGS, Ramos JV, Avelino FVSD. Conhecimento de enfermeiras sobre evento adverso na atenção primária à saúde. Revista enfermagem atual [internet]. 2019 [acesso em 05 Nov 2020]; 89(27):01-07. Disponível em:

https://revistaenfermagematual.com.br/index .php/revista/article/view/459/475.

5. Lemos GC, Azevedo C, Bernardes MFVG, Ribeiro HCTC, Menezes AC, Mata LRF. A Cultura de Segurança do Paciente no
Âmbito da Enfermagem: Reflexão Teórica. Revista de Enfermagem do Centro-Oeste Mineiro. [internet]. 2018 [acesso em 14 Nov 2020]; 8:e2600: 01-10. Disponível em: http://www.seer.ufsj.edu.br/index.php/recom /article/view/2600/1880.

DOI: https://doi.org/10.19175/recom.v7i0.2600.

6. Machado NCB, Morais EM, Fontana RT, Rodrigues AP, Barros M, Krebs EM. Percepção de discentes, docentes e técnicos em enfermagem a respeito dos eventos adversos. Rev. Enferm. UFSM [internet]. 2020 [Acesso em 29 Out 2020]; 10e:1-18. DOI:

https://doi.org/10.5902/2179769233486.

7. Alves MFT, Carvalho DSD; Albuquerque GSCD. Motivos para a não notificação de incidentes de segurança do paciente por profissionais de saúde: revisão integrativa. Ciênc. saúde coletiva [Internet]. 2019 [acesso em 29 Out 2020 ]; 24(8):2895-908. Disponível em: http://www.scielo.br/scielo.php?script=sci_a rttext\&pid=S1413-

$81232019000802895 \& \operatorname{lng}=\mathrm{en}$.

DOI: http://dx.doi.org/10.1590/141381232018248.23912017.

8. Maia CS, Freitas DRC, Gallo LG, Araújo WN. Notificações de eventos adversos relacionados com a assistência à saúde que levaram a óbitos no Brasil, 2014-2016. Epidemiol. Serv. Saúde [Internet]. 2018 [acesso em 05 Nov 2020]; 27(2):e2017320. Disponível

em: http://www.scielo.br/scielo.php?script=sci_a rttext\&pid=S2237-

$96222018000200308 \& \operatorname{lng}=\mathrm{en}$.

DOI: http://dx.doi.org/10.5123/s167949742018000200004.

9. Oliveira RM, Bandeira ES, Silva CR, Soares AML, Fonteles DB, Barboza FBM. Tomada de decisão de enfermeiros frente a incidentes relacionados à segurança do paciente. Cogitare Enfermagem [internet]. 2016 
[acesso em 05 Nov 2020]; 21(3):01-10. Disponível em: https://revistas.ufpr.br/cogitare/article/view/ 45683/pdf.

10. Ganong LH. Integrative Review of Nursing Research. Res Nursing Health, 1987. Febr; 10(1):1-11. DOI: 10.1002 / nur.4770100103.

11. Souza MT, Silva MD, Carvalho R. Revisão integrativa: o que é e como fazer. einstein (São Paulo). 2010 [acesso em 24 Out 2020]; 8(1):102-6.

DOI: https://doi.org/10.1590/s167945082010 rw1134.

12. Santos CMC, Pimenta CAM, Nobre MRC. A estratégia pico para a construção da pergunta de pesquisa e busca de evidências. Rev Latino-am Enfermagem [internet]. 2007 maio-junho [acesso em 29 Out 2020]; 15(3):1-04. Disponível em: https://www.scielo.br/pdf/rlae/v15n3/pt_v15 n3a23.pdf.

13. Ponte

RF,

Pontes

KCJR. Academical. Academical Sistemas para Pesquisas Científicas Ltda. Chapecó, 2019. Disponível

em: https://www.academical.com.br [internet]. 2018 [Acesso em 26 Out. 2020] Disponível em:

https://app.academical.com.br/pesquisa/304/ referencias/exportar/2.

DOI: https://doi.org/10.5123/s1679-

49742018000200004.

14. Moher D, Liberati A, Tetzlaff J, Altman DG. The PRISMA Group (2009). Preferred Reporting Items for Systematic Reviews and Meta-Analyses: The PRISMA Statement. J Clin Epidemiol [Internet]. 2009 [acesso em 24 Out 2020]; 62(10):1006-12. DOI: 10.1016/j.jclinepi.2009.06.005.

15. Bardin L. Análise de conteúdo. São Paulo: Edições,
https://www.ets.ufpb.br/pdf/2013/2\%20Met odos\%20quantitat $\% 20 \mathrm{e} \% 20$ qualitat $\% 20$ $\%$ 20IFES/Livros\%20de\%20Metodologia/10 $\% 20-\% 20$ Bardin,\%20Laurence\%20$\% 20 \mathrm{An} \% \mathrm{C} 3 \%$ A1lise $\% 20 \mathrm{de} \% 20 \mathrm{Conte} \% \mathrm{C} 3$ $\%$ BAdo.pdf.

16. Beuren IM, Souza JC. Em busca de um delineamento de proposta para classificação dos periódicos internacionais de contabilidade para o Qualis CAPES. Rev Contab finanç [Internet]. 2008 [acesso em 24 Out. 2020]; Jan/Apr; 19(46):44-58. DOI: 10.1590/S1519-70772008000100005.

17. Marchon SG, Mendes Junior WV, Pavao ALB. Características dos eventos adversos na atenção primária à saúde no Brasil. Cad. Saúde Pública [internet]. 2015 [acesso em 24 Out 2020]; 31(11):2313-330. Disponível em:https://www.scielo.br/pdf/csp/v31n11/01 02-311X-csp-31-11-2313.pdf. DOI: http://dx.doi.org/10.1590/0102311X00194214.

18. Ternopolski CA, Baratieri T, Lenstck MH. Eventos adversos pós-vacinação: educação permanente para a equipe de enfermagem. Rev. Espaço para Saúde [internet]. 2015 [acesso em 24 Out 2020]; out/dez; Londrina 16(4): 109-19. Disponível em: http://espacoparasaude.fpp.edu.br/index.php/ espacosaude/article/view/388/13. DOI: http://dx.doi.org/10.22421/1517$7130.2015 \mathrm{v} 16 \mathrm{n} 4 \mathrm{p} 109$.

19. Bisetto-Linheira LH, Ciosak SI, Cordeiro TLR, Boing MS. Ocorrência de eventos adversos pós-vacinação em idosos. Cogitare Enferm. [internet] 2016 [acesso em 24 Out 2020]; Out/dez; 21(4):01-10 Disponível em: http://docs.bvsalud.org/biblioref/2016/12/82 7175/45682-190057-1-pb.pdf. DOI: http://dx.doi.org/10.5380/ce.v21i4.45682.

20. Oliveira ESA, Casarin ST, Coelin T, Soares DC. Cuidados prestados pela equipe de 
enfermagem no atendimento aos eventos adversos pós-vacinais. Rev. de Enfermagem da UFPI [internet]. 2016 [acesso em 24 Out 2020]; Abr-Jun; 5(2):32-40. DOI: https://doi.org/10.26694/reufpi.v5i2.5275.

\section{Pereira LB, Jobim L, Bueno D. Percepção} de Equipes de Saúde da Família sobre a notificação de eventos adversos a medicamentos. Saúde em Redes [internet]. 2018 [acesso em 24 Out 2020]; 4(3):49-61. DOI: $\quad$ https://doi.org/10.18310/2446$48132018 \mathrm{v} 4 \mathrm{n} 3.2207 \mathrm{~g} 298$.

22. Moura RS, Saraiva FJC, Santos RM, Santos RFM, Rocha KRSL, Virgem MRC. Eventos adversos ocasionados pela assistência de enfermagem: noticiados pela mídia. Revista de Enfermagem UFPE [Internet]. 2018 [acesso em 24 Out 2020]; 12(6): 1514-23. Disponível em: https://periodicos.ufpe.br/revistas/revistaenf ermagem/article/view/231266. DOI: https://doi.org/10.5205/1981-8963v12i6a231266p1514-1523-2018.

23. Bisetto LHL, Cubas MR, Malucelli A. A prática da enfermagem frente aos eventos adversos pós-vacinação. Rev. esc. enferm. USP [Internet]. 2011 [acesso em 24 Out 2020]; 45(5): 1128-34. Disponível em: http://www.scielo.br/scielo.php?script=sci_a rttext\&pid=S008062342011000500014\&lng=en.

DOI: https://doi.org/10.1590/S008062342011000500014 .

24. Alves H, Domingos LMG. Manejo de eventos adversos pós-vacinação pela equipe de enfermagem: desafios para o cuidado. Rev. Enfermagem UERJ [internet]. 2013 [acesso em 24 Out 2020]; Rio de Janeiro, out/dez; 21(4):502-7. Disponível em: https://www.e-

publicacoes.uerj.br/index.php/enfermagemu erj/article/view/10022.
25. Salvador PTC, Martins CCF, Alves KYA, Costa TD, Santos VEP. Análise de vídeos do youtube sobre eventos adversos em saúde. Rev Min Enferm. REME [internet]. 2014 [acesso em 24 Out 2020]; out/dez; 18(4): 830-37. DOI: $10.5935 / 1415-$ 2762.20140061.

26. Bisetto LHL, Ciosak SI. Análise da ocorrência de evento adverso pós-vacinação decorrente de erro de imunização. Rev. Bras. Enfermagem [Internet]. 2017 [acesso em 24 Out 2020]; Fev; 70(1): 87-95. Disponível

em: http://www.scielo.br/scielo.php?script=sci_a rttext\&pid=S0034-

$71672017000100087 \& \operatorname{lng}=$ pt.

DOI: https://doi.org/10.1590/0034-7167-20160034.

27. Melnyk BM, Fineout-Overholt E. Making the case for evidence-based practice. In: Melnyk BM, Fineout-Overholt E. Evidencebased practice in nursing \& healthcare: A guide to best practice. 2th ed. Philadelphia: Lippincot Williams \& Wilkins; 2005. p. 3-24. [acesso em Mar. 2021];

28. Silva MFB, Santana JS. Erros na administração de medicamentos pelos profissionais de enfermagem. Arquivos Catarinenses de Medicina [Internet]. 2018 [Acesso em 05 Nov 2020]; 47(4):146-54. Disponível em: http://www.acm.org.br/acm/seer/index.php/a rquivos/article/view/359.

29. Souza LM, da Silva MCS, Zavalhia SR, Coppola IS, da Rocha BP. Percepção de enfermeiros da Estratégia de Saúde da Família sobre segurança do paciente. J. nurs. Health [internet]. 2018 [acesso em 29 Out 2020]; 8(2):01-16. Disponível em: file:///C:/Users/Admin/Desktop/Downloads/ 12721-50021-1-PB\%20(1).pdf. DOI: HTTPS://DOI.ORG/10.15210/JONAH.V8I2 .12721 . 
Aprovado: 2021-03-31

30. Barboza TC, Guimarães RA, Gimenes FRE, Silva AEBC. Retrospective study of immunization errors reported in an online Information System. Rev. Latino-Am. Enfermagem [Internet]. 2020 [acesso em 14 Nov 2020]; 28:e3303. Disponível em: http://www.scielo.br/scielo.php?script=sci_a rttext\&pid=S0104$11692020000100352 \& \operatorname{lng}=$ en.

DOI:https://doi.org/10.1590/15188345.3343.3303.

31. Lima SMS, Agostinho M, Mota L, Príncie F. Perceção dos profissionais de saúde das limitações à notificação do erro/evento adverso. Revista de Enfermagem Referência [internet]. 2018 [acesso em Nov 2020]; out./nov./dez; Série IV(19) - Disponível em: http://www.scielo.mec.pt/pdf/ref/vserIVn19/ serIVn19a11.pdf.

DOI: https://doi.org/10.12707/RIV18023.

32. Nogueira JWS, Rodrigues MCS. Comunicação efetiva no trabalho em equipe em saúde: desafio para a segurança do paciente. Ver. Cogitare Enfermagem [internet]. 2015 [acesso em 05 Nov. 2020]; 20(3): 636-40. Disponível em: https://revistas.ufpr.br/cogitare/article/view/ $40016 / 26245$.

33. Pires DEP, Machado RR, Soratto J, Scherer MA, Gonçalves ASR, Trindade LL. Cargas de trabalho da enfermagem na saúde da família: implicações no acesso universal. Rev. Latino-Am. Enfermagem [internet]. 2016 [acesso em 05 Nov 2020]; 24:e2677. Disponível em: https://www.scielo.br/pdf/rlae/v24/pt_01041169-rlae-0992-2682.pdf. DOI: http://dx.doi.org/10.1590/15188345.0992.2682.

Submissão: 2020-12-03 